Usage, Usability, and Utility of 3D City Models, 02005 (2012)

DOI: $10.1051 / 3 \mathrm{u} 3 \mathrm{~d} / 201202005$

(c) Owned by the authors, published by EDP Sciences, 2012

\title{
An ontology of 3D visualization techniques for enriched 3D city models
}

\author{
C. Métral, N. Ghoula and G. Falquet \\ Centre universitaire d'informatique, University of Geneva, 7 route de drize, \\ 1227 Carouge, Switzerland
}

\begin{abstract}
Models, which represent in 3 dimensions the geometric elements of a city, are called 3D city models. Those models are used for an intended wide range of applications beyond mere visualization. Such uses are made possible by enriching the geometrical aspects with urban or environmental data that cannot be perceptible to a user without a visual abstraction. Using such models implies interacting with them in order to perform specific tasks related to the visualized data. Those data are potentially heterogeneous in terms of types, scales, issues, etc. When creating an enriched 3D city model, the designer faces the problem of choosing the most relevant visualization techniques for those heterogeneous data that have to be displayed within the same scene. In this paper we address the problem related to the visualization of enriched 3D city models by proposing a formal representation of existing visualization techniques in the form of an ontology of 3D visualization techniques. We also show how this ontology can be used for computational reasoning and help for the selection of visualization techniques by checking if a technique is compatible with a dataset to display or to a technique already selected and used in the same scene.
\end{abstract}

\section{INTRODUCTION}

Since the 1960s, the imagination in the human computer interaction field has been focused on the idea of representing the real world in a virtual way. The concept of 3D interfaces has thus been explored and enriched continuously by new approaches of visualization. In particular for representing geometric elements of a city, we use 3D city models. For different usages, these models are enriched by information covering multiple issues.

Each type of enrichment data can be interpreted and transformed into a visual rendering leading to the design of different visualization techniques. The number of visualization techniques of enrichment information and virtual reality technologies is increasing. Nowadays, rendering a large amount of information within a 3D city model is becoming a less tedious task than it used to be. Regarding the number of available approaches, a designer of 3D city model should consider existing techniques in order to reuse them and reduce costs instead of starting from scratch. In order to collect the necessary knowledge about available visualization techniques for 3D display in a given case, a designer is obliged to go through a large amount of scientific or industrial documentation. This documentation can have different types going from detailed specifications to only general information. This variety in the description of visualization techniques cannot fulfill the information need of a 3D designer.

Some researchers have realized the fact that "no author or group of authors has written a comprehensive and authoritative text on the subject, despite a continuing and rich set of research findings, prototype systems, and products" [1] (Jim Foley in the foreword of the book "3D User Interfaces"). For this purpose, [1] had the initiative of writing about the concept of 3D user interfaces in order to give a large literature reviews and a set of examples and guidelines to understand this

This is an Open Access article distributed under the terms of the Creative Commons Attribution License 2.0, which permits unrestricted use, distribution, and reproduction in any medium, provided the original work is properly cited. 
research field. Learning about this field is not the only way for an advanced 3D designer to deal with the problems of offering effective and useful 3D displays. This domain is quite rich and large, so it is time consuming to be aware of all the novelties that may be useful in a particular case to display an amount of heterogeneous information (data sets) that have to be visualized together. This is why we propose an ontology driven knowledge base that allows a designer to get all the information he needs about the visualization techniques that are relevant for his/her context. Building this ontology of 3D visualization techniques relies on defining a formal representation of the different concepts related to $3 \mathrm{D}$ visualization techniques and their evaluation. In this paper we address the problem related to the visualization of enriched 3D city models by:

- proposing a formal representation of existing visualization techniques, in the form of a knowledge base of $3 \mathrm{D}$ visualization techniques that can be used for computational reasoning;

- representing and collecting usability evaluation results for each of the $3 \mathrm{D}$ visualization technique when available;

- defining rules about visualization techniques used in the same scene or about the compatibility of a technique with a data set to display;

- express user needs in terms of ontological tasks such as subsumption computation or logical query evaluation.

\section{STATE OF THE ART}

\subsection{Enriched 3D city models}

Models that represent in 3 dimensions the geometric elements of a city are called 3D city models. A large number of 3D city models have been recently created or are in the process of creation, for an intended wide range of applications beyond mere visualization. CityGML [2] is the first standard related to $3 \mathrm{D}$ city models. CityGML attempts to provide a description of $3 \mathrm{D}$ elements like relief, buildings, traffic infrastructure, water bodies, vegetation or city furnitures with their geometry, topology (spatial properties), semantic properties and relevant attributes. The attributes cover classification, function and actual usage of an object. Those objects have been identified to be either required or important in many different application areas. CityGML is also extendable to adapt to the requirements of specific applications. Such extensions have been defined for different issues such as noise, with noise immissions added to buildings, roads or railways [2]. We thus obtain 3D city models where the geometrical aspects have been extended with urban or environmental data that can be called enrichment data.

If visualization is one of the main uses of enriched 3D city models it is not the only one. The EU COST Action TU0801 considers 3D city models - semantically enriched with the knowledge underlying in data and models related to urban issues - as potential planning and decision-making tools whose assessment has still to be processed [3]. The use of enriched 3D city models for helping decision making in the urban domain is related to tasks performed by the user of such models and for which he/she has to navigate in or over the 3D model. Typical tasks include evaluation of urban projects in terms of quality of life (including visual aspects), evaluation of the impact of projects on the urban landscape and on other factors. Such tasks imply the visualization of data that can originate from different fields like transport, construction, air quality, noise, etc.; be of different kinds such as quantitative measures of noise or qualitative soundscapes; take different forms, from structured data provided by geographical information systems to unstructured documents; have different scales (city as a whole, buildings, etc.); be not directly georeferenced (legal text for example) although they have a spatiotemporal coverage. Although those data have to be represented with all their characteristics (type, georeferencing, urban or environmental field to which they are issued, etc.) their visualization can use techniques used in 3D virtual environments. 


\subsection{Representation of 3D visualization techniques}

Gathering or accessing multiple information and enrichment data about 3D city models is an important task. Many approaches and systems are available for seeking and organizing enrichment information [4]. Researches have been driven in order to make a best use of this enrichment knowledge by means of proposing new 3D visualization techniques [1]. Visualization techniques for 3D virtual environments are extensively used in simulation environments, video games, tutoring systems, etc. In the context of an enriched 3D city model, visualization techniques define a visual rendering of enrichment data allowing the user to explore and manipulate a large set of information.

Nevertheless only a few authors have attempted to classify these techniques to compare and evaluate them. Many documentations are focused on the description of new interaction techniques or software and hardware settings used in specific applications, most of them presenting also user studies for experimental evaluation. However, few works have been driven and can be found in literature trying to formalize the description of 3D User Interfaces [5]. For modeling and organizing knowledge about a specific domain, there are different existing approaches. Ontologies are lately one of the most used tools to establish a knowledge management solution [6]. These are engineering artifacts [7] that need to be constructed, refined, updated and integrated based on knowledge from domain experts. Formal ontologies are based on logical formalisms, they offer a possibility for human users and software agents to share and use knowledge.

Different classifications, terminologies, taxonomies or ontologies have been defined in the field of visualization, with different aims. [8] presented the design of a visualization ontology, which aims at providing more semantics for the discovery of visualization services. This ontology treats the problem of designing visualization techniques from an application level and processing but it does not detail the specifications of these techniques. The Top Level Visualization Ontology (TLVO) defined by [9] aims at providing a common vocabulary to describe visualization data, processes and products. Based on an analysis of visualization taxonomies and on recent work in visualization ontologies, [10] propose some modifications to the TLVO in order to better represent the visualization process and data models. For the purpose of describing visualization techniques and their evaluations, this ontology is not the best choice because it does not represent all the required concepts; for instance, there is no representation of $3 \mathrm{D}$ visualization, neither the evaluation of these techniques nor the representation of the usage context. [11] aims to developing a unifying ontology, which is applicable in visualization systems. By systematically reviewing existing models and classifications, they found in particular that most visualization knowledge is stored informally in terminologies and taxonomies - thus being not directly usable for computational reasoning - while the few existing visualization ontologies do not sufficiently represent existing domain knowledge and, furthermore, are not accessible to public. More recently, [12] have created a visualization ontology to support a recommendation system for the selection of visualization components.

In our case, we aim at organizing and formally representing knowledge about visualization techniques for enriched 3D city models. Existing ontologies cannot be entirely reused as they focus on the visualization process and not on the required entities for an effective representation and reuse of visualization techniques. By means of the proposed ontology of visualization techniques we intend to: (i) propose a formal representation for describing existing visualization techniques, (ii) define rules for combining and reusing these techniques, and (iii) show how selection tasks, such as checking if a technique is compatible with a dataset to display, can be expressed in terms of ontological tasks such as subsumption or logical query evaluation.

\subsection{Evaluation of 3D visualization techniques}

When designing an enriched 3D city model, the designer faces the problem of choosing the most relevant visualization techniques for viewing the geometric and the enrichment data. This means that 
the selected techniques must at least be able to display the desired information (effectivity); efficiently support the user task(s); not negatively interfere with each other (e.g. by hiding information). Given the vast amount of visualization techniques that have been developed over the last decades, and the variety of visual contexts, user tasks, and data types, the selection of relevant visualization techniques is far from trivial. When the evaluation results are publicly available they can be used to help selecting a technique.

An example of such a case is [13], who proposes two new visualization techniques for enhancing the visibility of road labels in 3D navigation maps, the expected enhancement being defined relatively to the baseline approach (which is used in almost all existing navigation systems such as Google Earth) and confirmed by a user study. In fact we usually don't have such evaluation results. On the contrary many visualization techniques are used in 3D geographic information systems (3D GIS) by many users and for many tasks without any evaluation. While not formally assessed the utility of such visualization techniques still exists. Visualization techniques used in 3D video games can also be considered as some of them are close to 3D enriched city models. It is the case with Simcity, for example, which is a citybuilding and urban planning simulation computer game [14]. But, in the case of video games if intensive evaluations of the 3D visualization techniques used have been performed, their results are usually kept secret. [15] has investigated the domain of abstract information integration into interactive 3DVEs. He highlighted that there are many different approaches in order to help the user perform specific tasks.

In order to be able to decide about the usability of a visualization technique, designers use evaluation methods. Most of the evaluation methods have been originally conceived for 2D usability evaluation and then they were adapted and extended to support 3D user interfaces evaluation [16]. The number of evaluation methods of 3D user interfaces usability is continuously growing. There are different kinds of evaluation methods such as heuristic evaluation [17] or formative evaluation [18] etc. Formalizing the representation of 3D visualization techniques for enrichment data is not enough for using the resulting knowledge base. To define and decide about the usability of such techniques in real cases we should collect and organize all the information related to their evaluation. This brings us to also define a formal representation of the concept of usability evaluation for 3D user interface in the case of enriched 3D city models. First, we have to be able to classify evaluation methods and identify their metrics. Second, we have to take into account the context and tasks of the evaluation. Which leads to the fact that the generality of the evaluation results depends on the generality of the context. This is why we also need to consider formalizing the context representation [19] and task descriptions [20].

\section{METHODOLOGY}

We address the problem related to the visualization of enriched $3 \mathrm{D}$ city models by proposing a framework in order to help a designer of an enriched 3D city model to choose the best visualization techniques for the data to visualize, the context in which this visualization will take place, and the task that will be performed by the user. The proposed framework comprises an ontology of 3D visualization techniques, a knowledge base containing the description of specific techniques, and selection tools that rely on this knowledge base. An ontology-based approach enables not only a formal representation of existing but scattered knowledge about visualization techniques but also computational reasoning based on different criteria. These criteria can be related to the usability evaluation of a technique, as evaluation results will be stored in the knowledge base.

The research methodology we used to create the ontology and the knowledge base consists in the following main phases:

1. Conceptualization. We obtained a conceptualization of 3D visualization techniques by collecting, adapting, and extending models, existing ontologies and taxonomies about 3D visualization techniques described in the literature. 


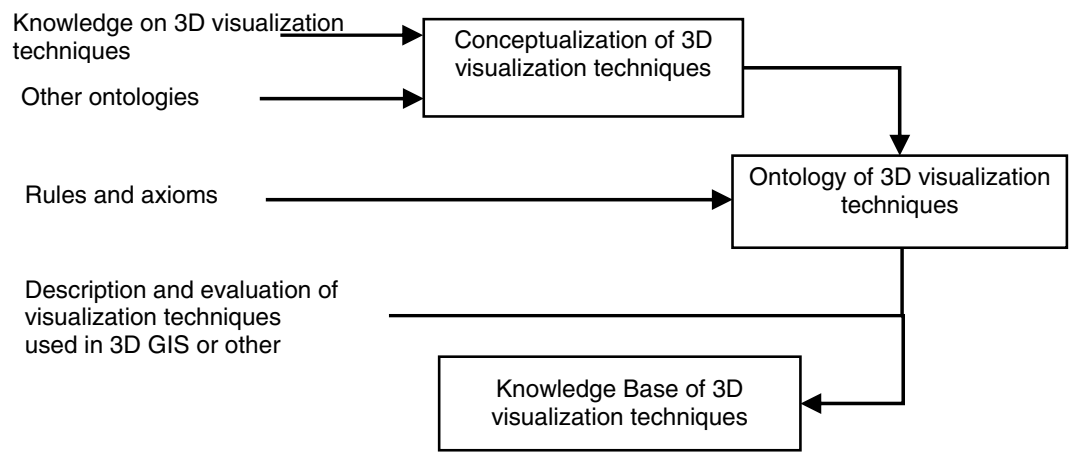

Figure 1. Methodology of the creation of an ontology of 3D visualization techniques and of the knowledge base associated.

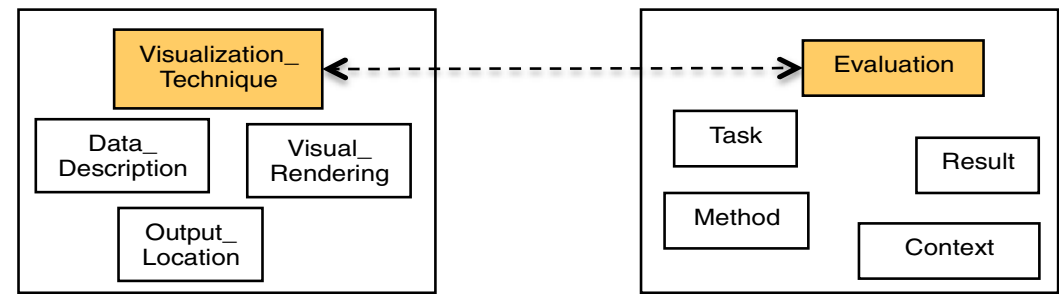

Figure 2. Specification of the interacting modules of the ontology for representing visualization techniques.

2. Formalization. We formalized the obtained conceptualization in the OWL2 ontology language. More precise knowledge (for instance, generic data/technique compatibility) was introduced as ontology axioms.

3. Extension towards evaluation. The ontology has been complemented with a formalization of evaluation techniques directly drawn from human-computer interaction reference literature.

4. Populating the knowledge base. To obtain a knowledge base of visualization techniques we analyzed formal and informal descriptions and evaluations of 3D visualization techniques used (or proposed) in 3D GIS, 3D video games, and other applications.

\section{ONTOLOGY OF 3D VISUALIZATION TECHNIQUES}

\subsection{Modules of the ontology}

The design of the ontology is based on our study of the issue of visualization of enrichment information. In the section 2.3 we have identified that the use of visualization techniques representations depends also on their evaluation based on a context and tasks. Thus, as expressed in Figure 2, two modules compose this ontology.

The first module is related to the representation of 3D visualization techniques. The second module focuses on describing the evaluation of those techniques with regards to a specific or generic context related to a $3 \mathrm{D}$ model and a set of different tasks that a user can perform.

\subsection{Visualization techniques module}

This section describes the main concepts used to represent visualization techniques in the ontology. They are named Visualization_Technique and Data_description as each visualization technique applies to data that are specific to it and that need to be described. 


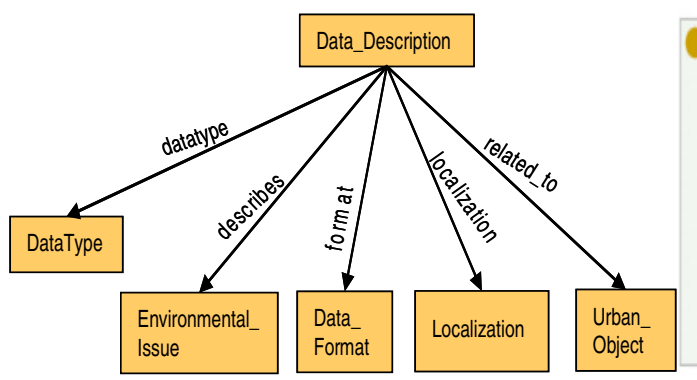

(datatype some DataType)
and (describes some
Environmental_Issue)
and (format some Data_Format)
and (localization some Localization)
and (related_to some Urban_Object)
and (datasize some long)
and (datavalue some Literal)

Figure 3. Specification of the Data_Description concept (in graph form on the left, in OWL [22] on the right).

\section{Data_Description concept}

The description of a data includes the following elements:

- a data type, which can be, for example, a text, a string, an audio or video element, a scalar, a vector or a combination of those elements [21];

- a data format: describes the extension of the files containing the data, such as jpg, pdf, or xml, $\ldots$;

- the environmental issue to which it is related the enrichment information: air quality, noise, ...;

- the urban object(s) to which it is related, if any, ...;

- a (geo)localization in the 3D model, which can be in the form of coordinates, of a geoname, object related or another type of coverage;

The description of a data is represented in the ontology as a concept related by properties to the previous elements defined themselves as concepts.

A specific data object is represented as an instance of the Data_Description concept.

Visualization_Technique concept

A visualization technique is characterized by the following elements: a data description, an output location, a visual rendering, some visual attributes, a reference and example-s:

- Data Description. It represents the input to the technique and will be transformed into an output view based on different criteria of the metaphor of rendering and the output location manipulation and definition. Each data description can be used as input for many visualization techniques;

- Output Location. The output location describes the rendering criteria. It is specified in terms of: location (position and orientation), interaction and scale. It also represents the placement of the visual rendering regarding the object and the virtual environment: whether it is attached to the object, or displayed on the floor or on top of the user, etc.;

- Visual Rendering. The visual rendering represents an object abstraction before rendering it on the screen. It's about information that is viewable on the screen using a visualization technique [23];

- Visual attributes: they describe the visual aspects related to the technique: whether the data support is transparent or not, whether the size is fixed or dynamic, etc. [23];

- Reference: it is a link to a document describing the technique;

- Example: it is a link to a 3D prototype or an image capture of the technique's implementation.

\subsection{Evaluation module}

The purpose of enriched 3D city models evaluation is the identification of the usability of 3D techniques that are used to visualize enrichment data. Based on our study of the evaluation concept in section 2.3, we have managed to identify the majority of the related issues and concepts. Our interest for 


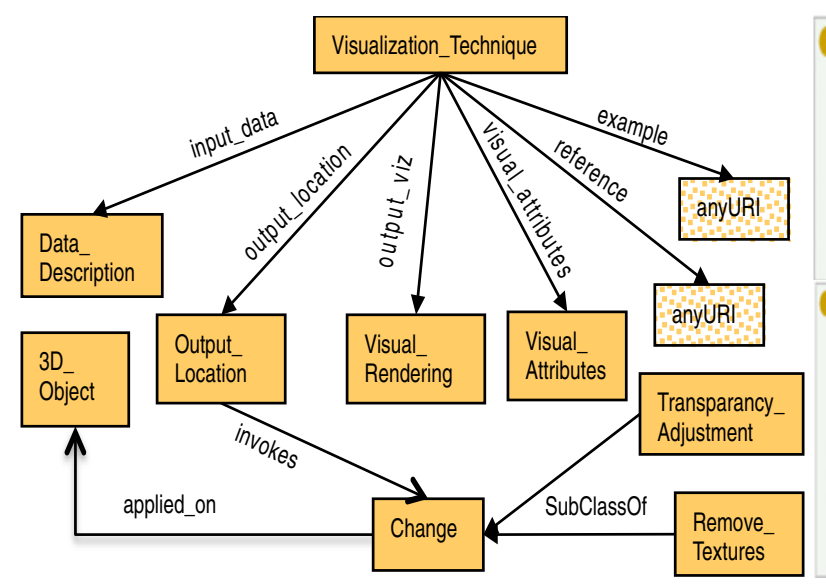

Oinput_data some Data_Description)
and (output_location some Output_Location)
and (output_viz some Visual_Rendering)
and (visual_attributes some Visual_Attributes)
and (example some anyURI)
and (reference some anyURI)
O(layout_interaction some Output_Location_Interaction)
and (layout_scale some Output__cale)
and (layout_shape some Output_Shape)
and (layout__ppace some Output_location_Space)
and (location some Output_Placement)
and (location_orientation some Output_Location_orientation)
and (location_position some Output__ocation__osition)
and (invokes some Change)

Figure 4. Specification of the Visualization_Technique concept (in graph form on the left, in OWL on the right).

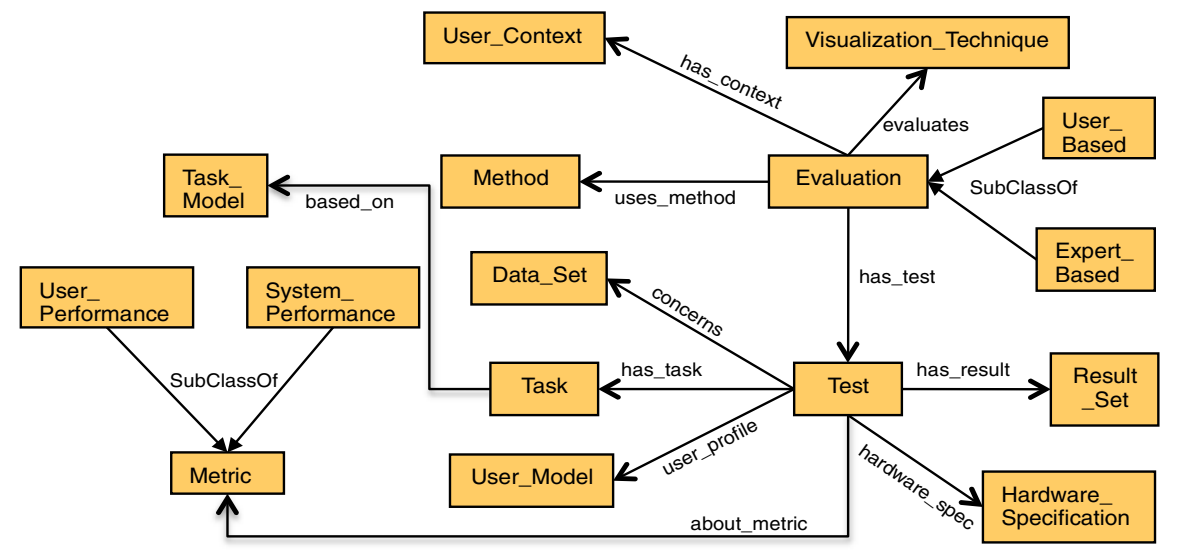

Figure 5. Specifications and representation of the Evaluation concept.

representing evaluations is in the same level of our interest for representing visualization techniques. Collecting information for describing techniques is not enough to offer a framework to integrate and reuse them. We need to have information about their usability in a context of a 3D city model. The access to the evaluations of 3D visualization techniques allows us to have a record about the usability evaluation's results for these techniques in a given context. Once we manage to gather these results and classify them by type (effectiveness metrics, effectivity metrics and user satisfaction metrics), we can run advanced processes in order to compare $3 \mathrm{D}$ visualization techniques and help a designer to decide about the usability of these techniques is his/her context.

Representing evaluations and storing results for each technique should be based on a definition of the relation between all the entities used in the evaluation process. For example one of these entities is the user context, which is a complex concept that needs to be represented in order to understand its criteria and be able to compare its instances between each other. We have designed and tested the following model for representing evaluations of 3D visualization techniques for enrichment data.

Each evaluation of visualization techniques measures aspects of their usability. These measures are related to methods of evaluation. Methods can measure different aspects having different categories of metrics such as system performance, which is a metric for measuring the performances of the 


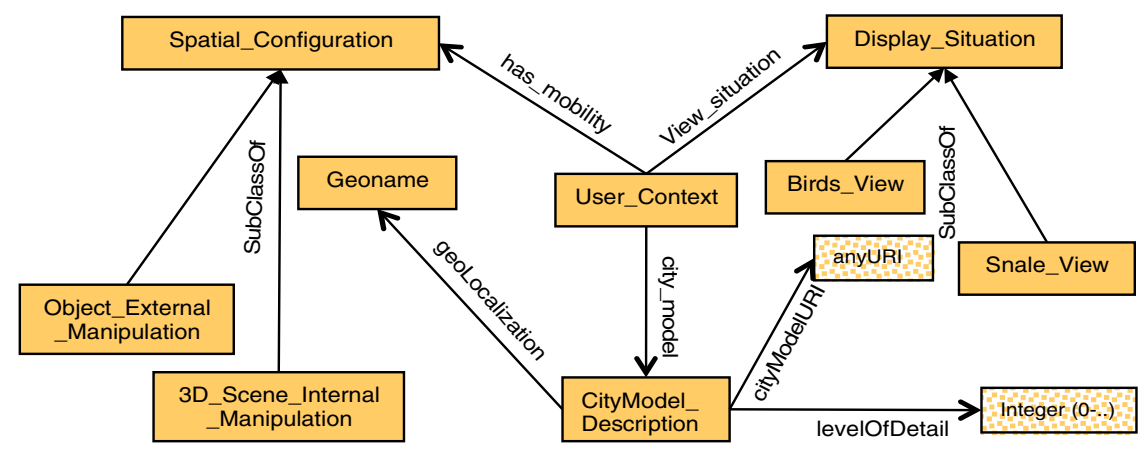

Figure 6. Specifications and representation of the User_Context concept.

visualization techniques in terms of optical distortion, network delay or calculations of visual rendering. We cannot compare evaluations of techniques unless they have been driven in the same context. The notion of user context in 3D city models is always present. We managed to extract different information about the context description from a corpus of scientific papers that we have collected and used from the beginning as a support for our methodology. To describe a user context we need to know about the special configuration of the user with regards to the 3D city model and its objects [24]. We need also information about the display situation of the 3D scene and also the level of details about the geometric objects (textured buildings, blocks, or shapes, etc.). A user context is related to a specific city or part of the city identified by a geoname. We consider the LOD as standard as it is a common criteria of 3D CM. Thus, we represent the concept of user context as follows.

\subsection{Compatibility rules for 3D visualization techniques}

Generic axioms are used for holding the internal consistency of the ontology in terms of visualization techniques description. For example, if we chose a 3D abstract visualization of the required data, we are not allowed to use an output location having as shape a 3D surface and a placement (the place where information are represented) attached to a terrain or a surface. The description of the output location must be coherent with the description of the visual rendering and localization associated to the enrichment data to display.

Determining compatibility rules for using visualization techniques of enrichment information in the same user context depends on different criteria and situations. For example, in the same localization in the 3D model, we may have three data related to the same building: (1) a short text (string type) describing the building's name, (2) a set of scalar values representing air quality, and (3) a set of scalar values representing noise at the same location. Two data have the same type so they could potentially be visualized using the same technique. But the user may be confused and take the information about air quality as noise level and vice versa. An incompatibility rule can avoid such confusion in allowing the same visualization technique being used only once. Another problem is related to possible occlusions. The representation of the label data could hide or be an obstacle for viewing the other data: thus, if the label is represented as a 3D text object it should be represented in a side or attached to the top of the building leaving enough space for representing the scalar values of noise and air quality.

\section{EVALUATION OF THE ONTOLOGY}

As stated by Uschold and King [25], an ontology must be evaluated against a given frame of reference. In our case the frame of reference is 1) the set of existing techniques, as described in the literature, and 2) a set of "competency questions" that correspond to typical user queries. The first evaluation step consists 

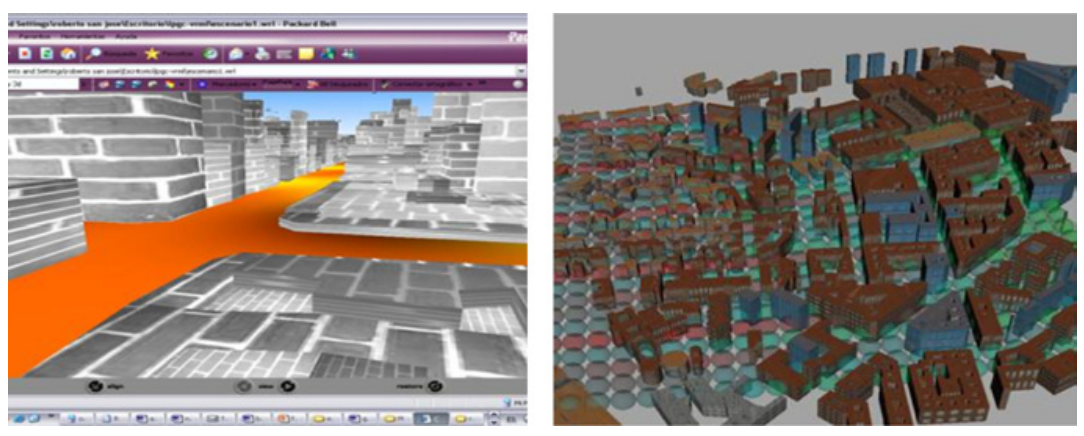

Figure 7. Two techniques (ColoredTextures on the left, ColoredBalls on the right) for representing air quality data [26].

in checking that typical visualization techniques can effectively be represented with the vocabulary provided by the ontology. More precisely, can we collect different 3D visualization techniques and effectively formalize them to produce a knowledge base of (evaluated) 3D visualization techniques. We have begun the evaluation process by selecting six techniques that we consider as representative and by describing them using the concepts formerly defined. We then defined several queries in order to select some techniques according to some criteria.

\subsection{Visualization techniques for air quality data}

The techniques described in this section issue from [26]. We have created instances of the class visualization techniques describing the criteria of these techniques and attached the data description to the air quality issue.

\subsubsection{ColoredTextures technique}

This technique displays numeric values, corresponding to air quality levels relative to car traffic, as colored textures put on the objects representing the streets in the model.

The user context is described by: street level for the display situation, a static method of rendering and buildings textured in a level of detail LOD1. It has as input some numeric values with a 2D location.

The ColoredTextures technique (named AirQuality_Scalar_WS_2D_ColoredTextures in the ontology) is represented as follows:

\subsubsection{ColoredBalls technique}

This technique displays scalar values, corresponding to air quality levels relative to car traffic, as colored spheres put on the objects representing the streets in the model. The user context is described by: street level or birds' eye view for the display situation, a static method of rendering and buildings non-textured with a level of detail LOD2.

This 3D visualization technique has as input some scalar values with a 3D location. The ColoredBalls technique (named AirQuality_Scalar_VS_3D_ColoredBalls in our ontology) is represented as follows:

These two visualization techniques are rendering enrichment information having different data types for the same 3D city model and with comparable contexts. These two techniques cannot be used together within the same 3D city model. They have a total occlusion because they use the same display space for the visual rendering (the colored spheres hiding the colored textures in the streets). 
Usage, Usability, and Utility of 3D City Models

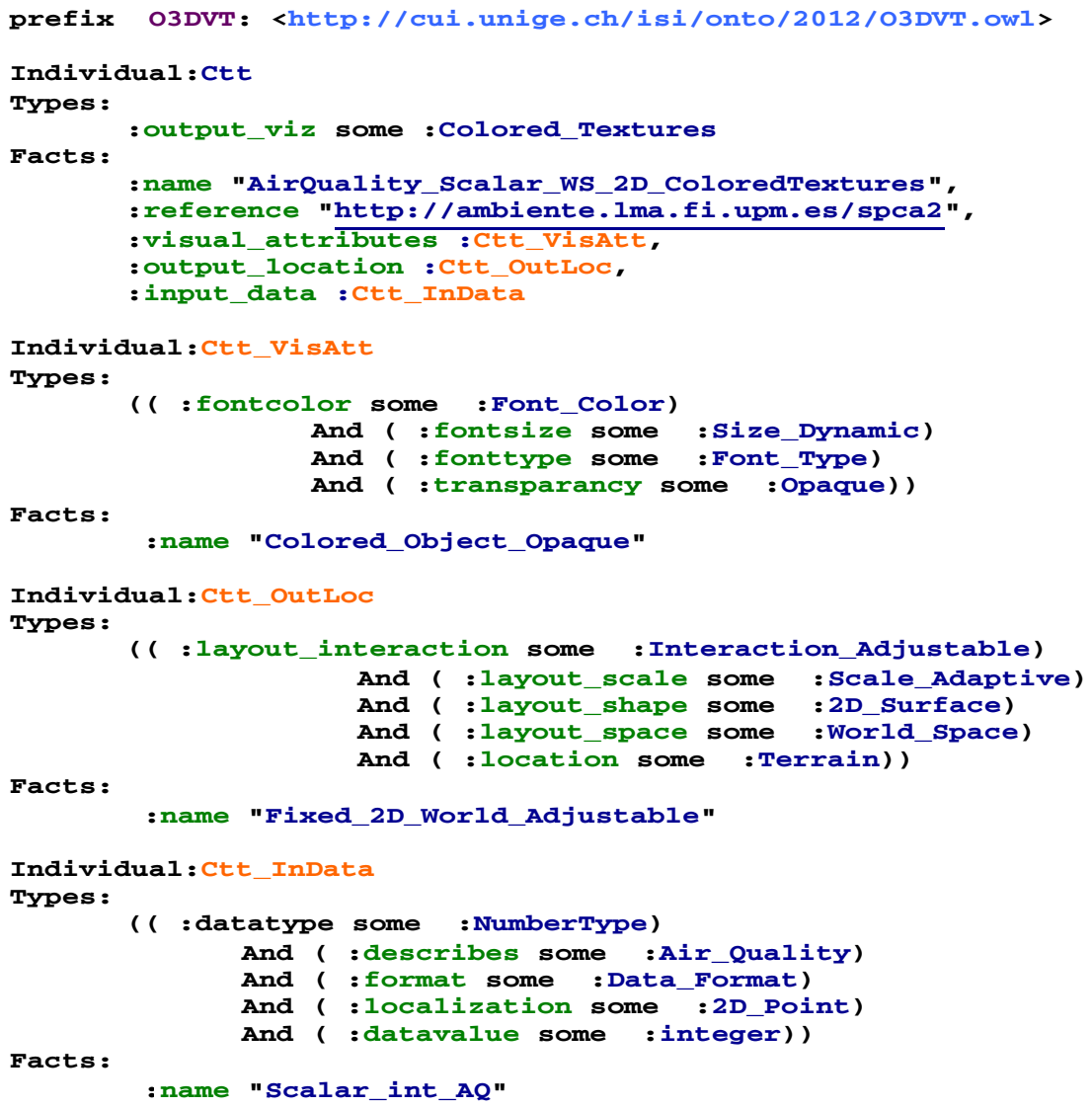

Figure 8. Representation in Manchester (Protégé [27]) notation of the ColoredTextures technique.

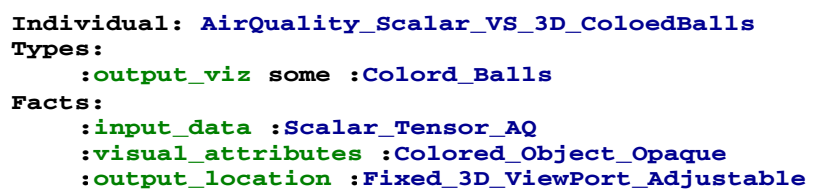

Figure 9. Representation in Manchester (Protégé) notation of the ColoredBalls technique.

\subsubsection{Examples of queries}

Queries can be performed to select some relevant visualization techniques from some criteria. Figure 11 gives examples of such queries. The first query (on the left) retrieves all described visualization techniques relevant for visualizing data in the field of air quality. The second query (on the right) retrieves all described visualization techniques (1) relevant for the visualization of numeric data in the field of air quality and (2) having a layout technique localized in a terrain as a 2D surface.

\subsection{Visualization techniques for enhancing the visibility of labels in 3D navigation maps}

The techniques described in this section issue from [13] and have been developed for enhancing the visibility of textual labels in 3D navigation maps as those labels can become occluded. Here is their 

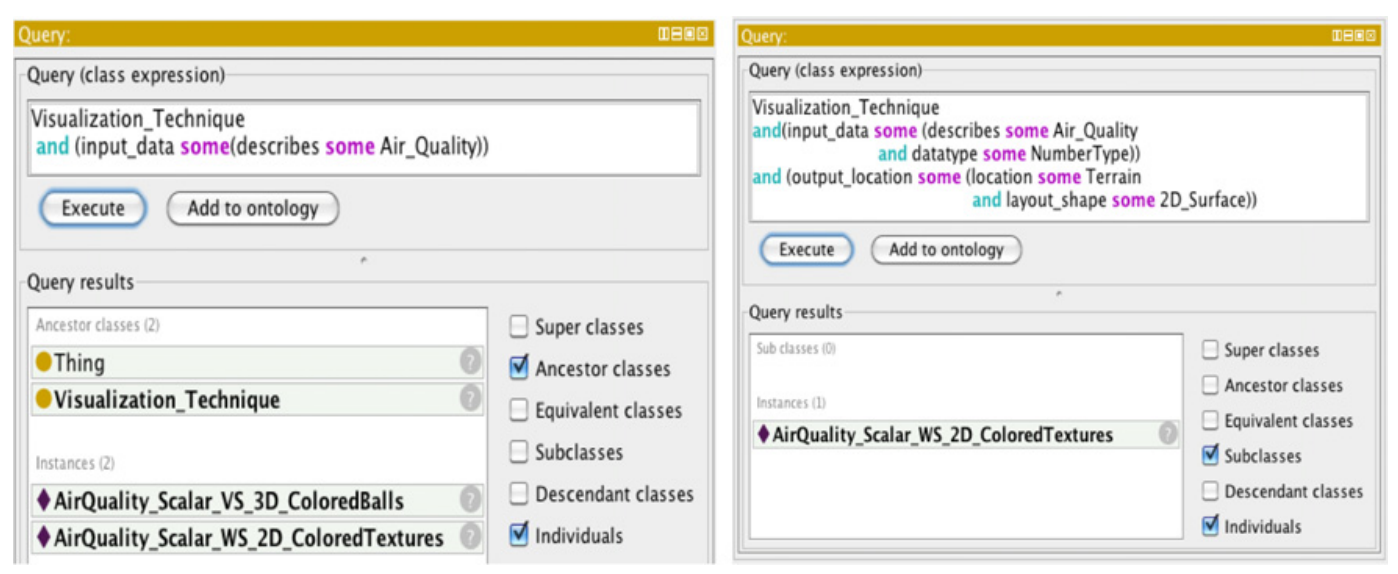

Figure 10. Querying the knowledge base to get all the techniques responding to some criteria (in OWL).
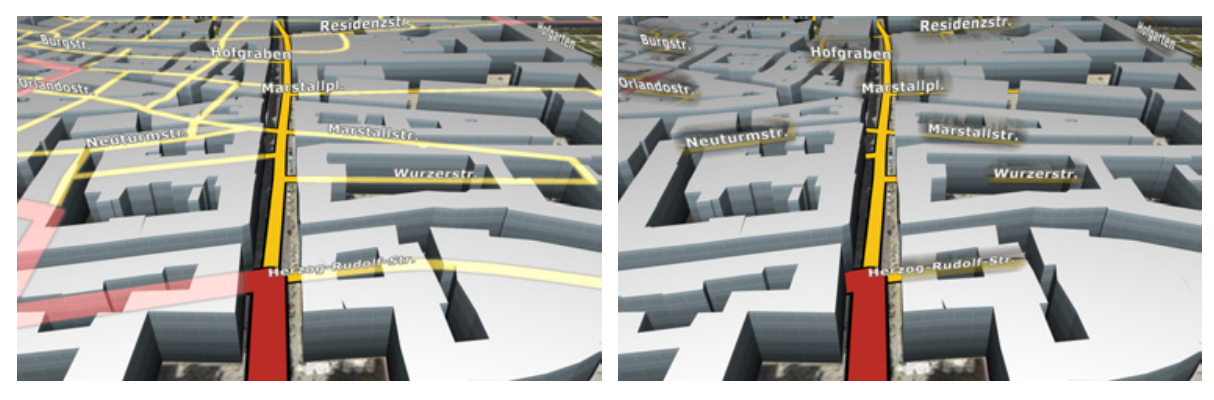

Figure 11. Two techniques (TransparencyLabelsAura on the left, GlowingRoads on the right).

description:

- TransparencyLabelAura technique: it lets the label shine through occluding objects;

- GlowingRoads technique: it lets the roads glow through the 3D environment.

For both techniques an evaluation has been performed; the user task is to follow an itinerary.

These two techniques use the same data, which is an individual of the "Data_Description" concept with a data type as a short text.

For TransparencyLabelAura technique, the labels create a transparent region in the occluding objects. This is represented as a change invoked by the output location used for this technique. For GlowingRoads technique the roads are glowing through the 3D world with a level of detail $\mathrm{LOD}=1$. The output location description of both techniques invokes changes in the geometry of the model.

There are two tests related to the evaluation of these techniques.

1. The first test is a benchmark evaluation aiming to evaluate the performance scalability and suitability for real-world scenarios. The benchmark results have concluded both techniques suitable for real-world scenarios.

2. The second test is user-based evaluation aiming to evaluate the usability, attractiveness and novelty of the approaches. This test is based on a user study method, which can be described as follows:

\section{User study = test of the prototype + AttrakDiff questionnaire.}


Usage, Usability, and Utility of 3D City Models

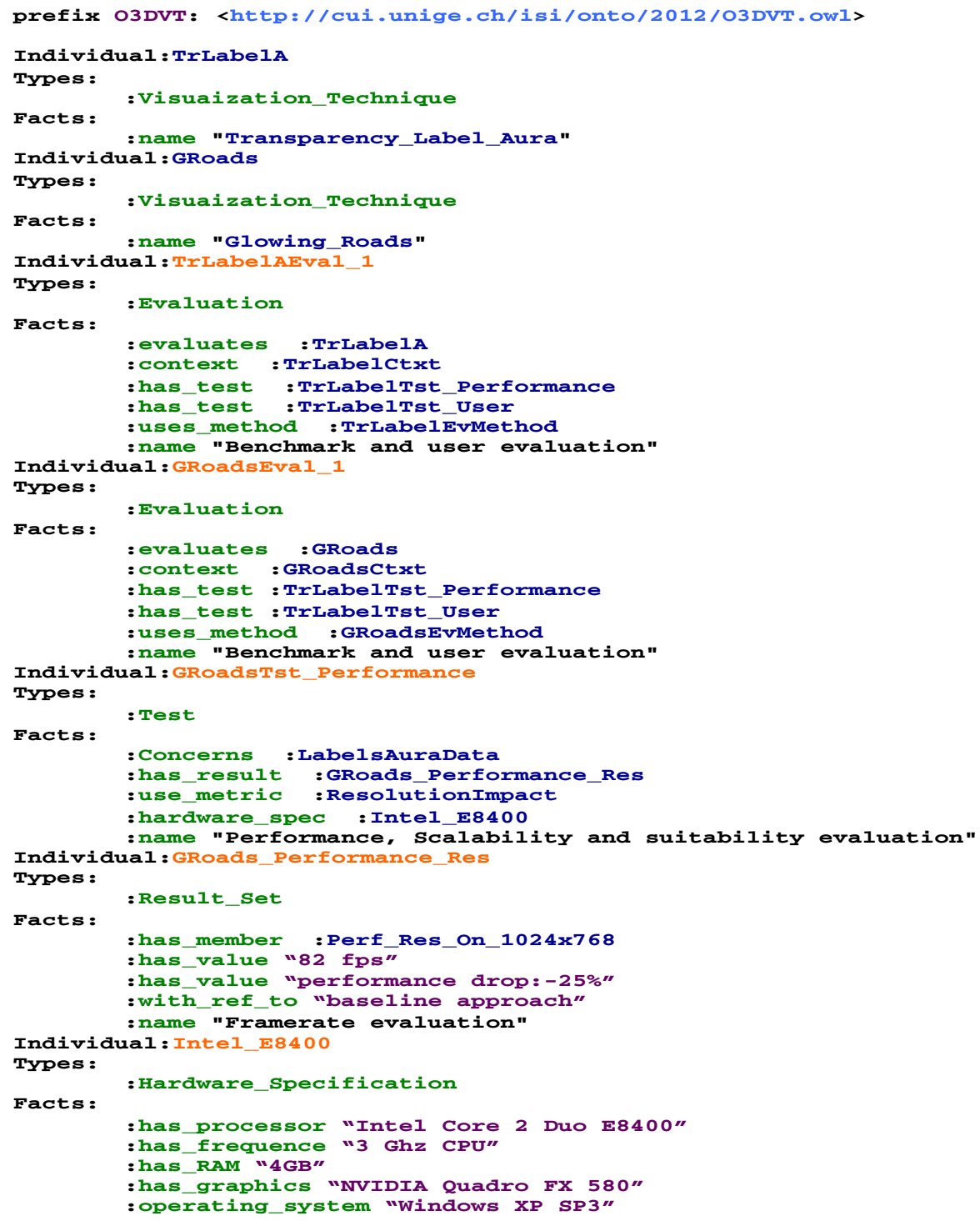

Figure 12. Excerpt from the description of TransparencyLabelsAura and GlowingRoads evaluation results [13].

The user evaluation results revealed that both methods improve significantly the usability and overall attractiveness [13].

\section{CONCLUSION AND FURTHER WORK}

In this paper we address the problem related to the visualization of enriched 3D city models, by proposing an ontology-based approach intended to help a designer of such models to choose the relevant visualization techniques in terms of usability. The ontology - and its associated knowledge base - are used to represent the various $3 \mathrm{D}$ visualization techniques associated to the potentially heterogeneous enrichment data. Such an approach (1) enables a common description of visualization techniques that can emanate not only from the urban field but from other fields such as 3D video games, (2) provides a formalized description that can be used to select automatically relevant techniques from the 
description of the data to display and from a set of specific criteria, taking in account the compatibility or incompatibility between techniques. We consider as hypothesis that the representation of the geometric objects in the 3D CM are already done. There is also a link between the 3D CM data format and the enrichment information format. The proposed techniques for a specific 3D CM should not be incoherent with its level of detail; otherwise we can state or activate the option that the visualization technique's implementation can induce changes in the 3D CM.

As it is difficult to know in advance the usability of a technique in a particular context, evaluations about the use of the visualization techniques are also represented in the ontology. It is not a simple task for a 3D UI designer to choose the right evaluation method for testing the usability of his visualization technique. At this stage of our research we are not intending to propose evaluation methods for each technique. However this is possible using our methodology. We need only to add domain-based rules about evaluations on top of the descriptions of evaluation approaches that are available in the knowledge base.

We began the evaluation of the ontology by formally representing several representative 3D visualization techniques. We plan to continue populating the knowledge base with more techniques. Although useful, evaluations about techniques may be inexistent, even for visualization techniques frequently used in 3D GIS. Thus we plan to perform by ourselves some evaluations that will be entered into the knowledge base. We also plan to define a user-friendly interface allowing the designer of a 3D visualization technique to describe his/her techniques using forms and to query the knowledge base by means of keywords and checklists.

The work described in this paper is part of the research "Designing and evaluating 3D knowledge visualization techniques for urban planning" funded by the Swiss "Secrétariat d'état à la recherche" (SER No C10.0150) in relation with the COST Action TU0801 "Semantic enrichment of 3D city models for sustainable urban development".

\section{References}

[1] D. A. Bowman, E. Kruijff, J. J. LaViola, and I. Poupyrev. 2004. 3D User Interfaces: Theory and Practice. Addison Wesley Longman Publishing Co., Inc., Redwood City, CA, USA

[2] OGC 08-007r1 (2008) OpenGIS City Geography Markup Language (CityGML) Encoding Standard. Gröger G., Kolbe T.H., Czerwinski A., Nagel C. (eds), Open Geospatial Consortium Inc, www.opengeospatial.org/standards/citygml, accessed February (2012)

[3] TU0801 (2008) Semantic Enrichment of 3D City Models for Sustainable Urban Development. Memorandum of Understanding, http://www.semcity.net/cms/index.php?page=reports, accessed February (2012)

[4] Falquet, Gilles and Métral, Claudine and Teller, Jacques and Tweed, Aidan Christopher, eds. 2011. Ontologies in urban development projects. Advanced information and knowledge processing, vol. 1. London: Springer

[5] H. Neale and S. Nichols: Designing and developing Virtual Environments: methods and applications. Visualization and Virtual Environments Community Club (VVECC) Workshop, Designing of Virtual Environments (2001)

[6] R. Dieng, O. Corby, A. Giboin, M. Ribière: Methods and tools for corporate knowledge management, International Journal of Human-Computer Studies, Vol. 51, Issue 3, September 1999, Pages 567-598, ISSN 1071-5819, 10.1006/ijhc.1999.0281

[7] N. Guarino, M. Carrara and P. Giaretta: Formalizing Ontological Commitment In Proceedings of National Conference on Artificial Intelligence (AAAI-94), Morgan Kaufmann, Seattle, pp. 560567 (1994) 
Usage, Usability, and Utility of 3D City Models

[8] A. S. Gao, J. Nick and F. R. Omer: Bringing semantics to visualization services. In: Advances in Engineering Software, Elsevier, Vol. 39, Issue 6, June (2008), pp. 514-520

[9] K.W. Brodlie, D.A. Duce, D.J. Duke, Visualization ontologies. Report of a workshop held at the National e-Science Centre, 7-8 April 2004, www.nesc.ac.uk/esi/download.cfm?index=1685, accessed Mars (2012)

[10] A. Morell Pérez, C. Pérez Risquet, J. Marx Gómez : An Enhanced Visualization Ontology for a Better Representation of the Visualization Process. In: ICT Innovation 2010, Communications in Computer and Information Science, M. Gusev and P. Mitrevski (Eds.), Springer-Verlag, Berlin Heidelberg, 2011, Vol. 83, Part 2, pp. 342-347

[11] M. Voigt, J. Polowinski: Towards a Unifying Visualization Ontology. Technical report, Institut für Software and Multimediatechnik, Technische Universität Dresden, Germany, March (2011), ISSN: 1430-211X

[12] M. Voigt, S. Pietschmann, L. Grammel, K. Meissner: Context-aware recommendation of visualization components. In: eKNOW 2012, the Fourth International Conference on Information, Process and Knowledge Management, pp. 101-109 (2012)

[13] M. Vaaraniemi, M. Freidank, R. Westermann: Enhancing the visibility of labels in 3D navigation maps. In: $7^{\text {th }} 3$ D GeoInfo conference, 16-17 May 2012, Québec, Canada

[14] SimCity (1989) Official SimCity website: http://www.ea.com/simcity, accessed February (2012)

[15] Bazargan Harandi, K. (2011). Abstract Information Visualization in Interactive 3D Virtual Environments: Conceptualization and Usability Evaluation. Thèse de doctorat, Université de Genève, 2011, no. SES 747, Edition SES - Université de Genève, ISBN 978-2-88903-005-7

[16] M. M. Sebrechts, J. V. Cugini, S. J. Laskowski, J. Vasilakis, and M. S. Miller: Visualization of search results: a comparative evaluation of text, 2D, and 3D interfaces. In Proceedings of the 22nd annual international ACM SIGIR conference on Research and development in information retrieval (SIGIR '99). ACM, New York, NY, USA (1999)

[17] J. Nielsen and R. Molich. 1990. Heuristic evaluation of user interfaces. In Proceedings of the SIGCHI conference on Human factors in computing systems: Empowering people (CHI '90), Jane Carrasco Chew and John Whiteside (Eds.). ACM, New York, NY, USA, 249-256, DOI=10.1145/97243.97281

[18] D. Hix, H. R. Hartson. Developing User Interfaces: Ensuring Usability Through Product \& Process, ISBN: 978-0-471-57813-0

[19] G. D. Abowd, A. K. Dey, P. J. Brown, N. Davies, M. Smith, and P. Steggles. 1999. Towards a Better Understanding of Context and Context-Awareness. In Proceedings of the 1st international symposium on Handheld and Ubiquitous Computing (HUC '99), Hans-Werner Gellersen (Ed.). Springer-Verlag, London, UK, 304-307

[20] Q. Limbourg and J. Vanderdonckt: Comparing Task Models for User Interface Design In The Handbook of Task Analysis for Human-Computer Interaction Mahwah: Lawrence Erlbaum Associates (2004)

[21] B. Shneiderman: The eyes have it: A task by data type taxonomy for information visualization. In: VL '96 : Proceedings of the 1996 IEEE Symposium on Visual Languages, Boulder, CO, USA, 03-06 September (1996)

[22] OWL Working Group (2009) OWL 2 Web Ontology Language: Document Overview. Publisher: W3C Recommendation, http: //www .w3.org/TR/ow12-overview/ accessed February (2012)

[23] Ed H. Chi: A Taxonomy of Visualization Techniques Using the Data State Reference Model. In: Proceedings of the IEEE Symposium on Information Vizualization 2000 (INFOVIS '00). IEEE Computer Society, Washington, DC, USA, pp. 69-75 (2000)

[24] F. Tyndiuk: Référentiels Spatiaux des Tâches d'Interaction et Caractéristiques de l'Utilisateur influençant la Performance en Réalité Virtuelle. Thèse de doctorat no 1269, Université Bordeaux $2,(2005)$ 


\section{European COST Action TU0801}

[25] M. Uschold and M. King. Towards a Methodology for Building Ontologies. In Workshop on Basic Ontological Issues in Knowledge Sharing, held in conjunction with IJCAI-95, Canada, (1995)

[26] R. San José, J. L. Pérez, R. M. González-Barras : 3D Visualization of Air Quality Data. In: Reliability and Statistics in Transportation and Communication Conference (RelStat'11), Riga, Latvia, 19-22 October (2011)

[27] Protégé (2005) The Protégé ontology editor and knowledge acquisition system, Stanford Center for Biomedical Informatics Research, http://protege.stanford. edu/, accessed February (2012) 